\title{
Die allergische bronchopulmonale Aspergillose (ABPA)
}

\author{
Allergic Bronchopulmonary Aspergillosis (ABPA)
}

\section{Zusammenfassung}

Die allergische bronchopulmonale Aspergillose (ABPA) ist die häufigste allergische bronchopulmonale Mykose bei Menschen. Die Diagnose des komplexen Krankheitsbildes basiert auf dem Nachweis definierter Kriterien. 5 klinische Stadien der ABPA werden unterschieden. Der Nachweis bzw. die Ausprägung der definierten Kriterien sind in den verschiedenen klinischen Studien unterschiedlich. Dies kann die Diagnose der ABPA erschweren. Insbesondere ist die Unterscheidung der ABPA in der Remission (Stadium II) vom allergischen Asthma mit Aspergillus-fumigatus-Sensibilisierung schwierig und kann ein wichtiges Problem sein. Die frühe Diagnose der ABPA in einem Stadium noch ohne persistierende Veränderungen der Bronchialwand und Destruktionen des Lungenparenchyms ist sehr wichtig, um die schweren Endstadien der Erkrankung zu verhindern. Die bisher in der Diagnostik benutzten, kommerziellen Allergenextrakte sind nicht optimal standardisiert, zeigen „batch to batch“-Variationen, was zu einem Mangel an Reproduzierbarkeit der Untersuchungen führen kann. Die Möglichkeiten und Grenzen der bisher durchgeführten diagnostischen Verfahren zur Diagnose der ABPA werden beschrieben. Die Produktion einer Anzahl rekombinanter Allergene des Aspergillus fumigatus und ihre In-vivound In-vitro-Evaluation bringt die frühe und präzise Diagnose der ABPA einen wichtigen Schritt weiter. Die für die präzise Diagnostik wichtigen rekombinanten Allergene sind jetzt für die Routinebestimmung im CAP-System erhältlich.

Kortikosteroide spielen eine zentrale Rolle in der Therapie der ABPA. Die Durchführung der Therapie in der Exazerbationsphase und die Langzeittherapie werden beschrieben und ebenso die Indikation für eine Therapie mit Antimykotika.

\section{Abstract}

Allergic bronchopulmonary aspergillosis (ABPA) is the most common allergic bronchopulmonary mycosis in humans. The diagnosis of the complex disease is based on defined criteria.

Five clinical stages of ABPA were proposed.

The extent of the defined criteria varies in the different stages, thus making diagnosis difficult. Particularly the discrimination of ABPA in remission stage (stage II) and allergic asthma with A. fumigatus-sensitisation may be an important problem. Early diagnosis in stages without persistant changes of bronchial wall and lung parenchyma is needed to prevent severe end stages of ABPA. The up to now widely used commercial (crude) allergen extracts for in vitro and in vivo diagnosis show batch to batch variation, insufficient standardization and lack of reproducibility. Potentials and limitations of routine diagnostic procedures in ABPA are described.

The production of a panel of recombinant allergens of A. fumigatus and their evaluation for in vivo and particularly in vitro use has brought an important step forward in the early and precise diagnosis of ABPA. A panel of recombinant allergens is now available for routine assay in CAP-System.

Glucocorticosteroids play a central role in therapy of ABPA. The approach in exacerbation phase and the long term therapy are described and also the indication for antimycotic drugs. 
Aspergillus (A.) gehört zu den ältesten und am häufigsten vorkommenden Pilzspezies. Diese Untergruppe der Ascomyceten wurde namentlich erstmals 1792 von Micheli beschrieben (aspergere $=$ zerstreuen, ausstreuen $)$.

Von den mehreren 100 verschiedenen Aspergillus-Spezies können einige, unter ihnen A.-fumigatus, A. flavus, A. niger und A. terreus, Krankheiten bei Mensch und Tier verursachen. Der Nachweis von Aspergillen im Sputum gesunder Menschen ist nichts Ungewöhnliches. Bei normaler Immunitätslage des befallenen Organismus werden die inhalierten Sporen durch Alveolarmakrophagen und die mukoziliäre Clearance eliminiert. Ganz anders ist die Situation bei immunsupprimierten Menschen, bei Allergikern oder auch bei besonders intensiver Exposition gegenüber pathogenen Aspergillen. Dabei können Aspergillen ihre pathogene Wirkung entfalten und sehr unterschiedliche Krankheitsbilder hervorrufen.

\section{Aspergillusassoziierte Erkrankungen des Respirationstraktes}

Die klinische Manifestation der Erkrankung ist abhängig vom Immunstatus des Wirtsorganismus, von bereits vorhandenen Schädigungen der Lungenarchitektur oder Störungen der mukoziliären Clearance sowie der Intensität der Exposition.

Aspergillusassoziierte Erkrankungen des Respirationstraktes können so unterteilt werden in invasive (Infektions-)Erkrankungen, allergische Erkrankungen und saprophytische Kolonisation (Tab.1).

Tab. 1 Aspergillusassoziierte Erkrankungen der oberen und unteren Atemwege

\begin{tabular}{ll}
\hline & Immunstatus \\
\hline $\begin{array}{l}\text { invasive pulmonale Aspergillose } \\
\text { chronische nekrotisierende Pneumonie }\end{array}$ & immunsupprimiert \\
\hline $\begin{array}{l}\text { invasive Aspergillus-Sinusitis } \\
\text { nicht invasive Aspergillus-Sinusitis }\end{array}$ & immunsupprimiert \\
\hline Aspergillom & nicht immunsupprimiert \\
\hline allergische Rhinitis & nicht immunsupprimiert/Kavernen \\
\hline allergische Aspergillus-Sinusitis & nicht immunsupprimiert/Atopiker \\
\hline $\begin{array}{l}\text { exogen allergische Alveolitis (EAA) } \\
\text { allergisches Asthma bronchiale }\end{array}$ & nicht immunsupprimiert/Atopiker/ \\
$\begin{array}{l}\text { (IgE vermittelt) } \\
\text { allergische bronchopulmonale }\end{array}$ & nicht immunsupprimiert immunsupprimiert/Atopiker \\
Aspergillose (ABPA) & Atopiker/CF/Bronchiektasen \\
\hline
\end{tabular}

\section{Allergische bronchopulmonale Aspergillose (ABPA)}

\section{Definition und Häufigkeit}

Die ABPA ist die häufigste bronchopulmonale Mykose bei Menschen. Sie wird üblicherweise durch Aspergillus fumigatus (A. fumigatus), seltener auch von anderen Aspergillus-Spezies verursacht.
Die ABPA wurde 1952 von Hinson u. Mitarb. erstmals als Krankheitsentität beschrieben [1] und in den folgenden Jahren zunächst als selten erachtet [2]. Heute wird die ABPA durch das zunehmende Verständnis und die verbesserten serologischen [3-6] und radiologischen [5,6] Untersuchungsmethoden besser definiert und auch etwas häufiger diagnostiziert. Bei Patienten mit Mukoviszidose wurde die ABPA erstmals 1965 beschreiben [7].

Bis heute stellt die ABPA jedoch ein klinisches Syndrom dar, dessen Diagnose durch den Nachweis einzelner typischer Kriterien, klinischer, laborchemischer und radiologischer Art (siehe unten) gestellt werden muss. Dies kann in weniger akuten Krankheitsstadien schwerfallen. Moderne Methoden der Molekularbiologie mit der Möglichkeit der Produktion rekombinanter Allergene und ihre Anwendung in der Diagnostik der ABPA eröffnen uns hier neue Möglichkeiten [4]. Wir werden in diesem Artikel später genauer darauf eingehen.

Aus Deutschland und Mitteleuropa liegen keine Angaben zur Prävalenz der ABPA vor. Untersuchungen aus den USA ergaben bei unausgewählten Asthmatikern eine Prävalenz von 7 bis $14 \%$. Bei Patienten mit steroidpflichtigem Asthma und A.-fumigatusSensibilisierung soll nach englischen Angaben der Anteil 15 bis $22 \%$ betragen.

Eine weitere prädisponierte Gruppe neben den Asthmatikern sind Patienten mit Mukoviszidose (zystische Fibrose $=C F$ ). Hier wird die Prävalenz unter den Patienten mit A.-fumigatus-Sensibilisierung mit bis zu $23 \%$ angegeben [7].

Die Diagnose der Erkrankung, die sich bereits im Kindesalter, aber auch im Jugendlichen- oder Erwachsenenalter manifestieren kann, wird häufig erst Jahre nach Beginn der Erkrankung festgestellt. Insbesondere wegen des potenziell schweren, progredienten Verlaufes ist eine frühzeitige Diagnosestellung wichtig, da sie unbehandelt zu schwerer Destruktion der Lunge führt.

\section{Pathomechanismen}

Die Krankheitsentität ABPA ist das Resultat immunologischer Entzündungsreaktionen im Bereich der Bronchien und dem umgebenden Lungenparenchym.

Immunologisch aktiv sind im Bronchialbaum mehrerer Antigene, die von A. fumigatus freigesetzt werden. Die Aspergillen wachsen in mukösen Pfröpfen in den Atemwegen von Asthmatikern und Mukoviszidose-Patienten, Mycelien wachsen auch in der Schleimhaut. Die immunologisch aktiven Substanzen, die vom Wirtsorganismus produziert werden, umfassen IgE-, IgGund IgA-Antikörper, die in die bronchiale Mukosa und ins Bronchiallumen gelangen. Dieses Nebeneinander von Antigenen und den reaktiven Produkten des Wirtsorganismus in den Bronchien und dem umgebenden Lungenparenchym resultiert in zumindest zwei Typen einer allergischen Reaktion: erstens die IgE-vermittelte Reaktion (Typ I nach Coombs und Gell), die Mastzellen und zusätzlich eosinophile Leukozyten, T-Lymphozyten und Makrophagen involviert. Diese werden durch Freisetzung von Mediatoren rekrutiert. Weiter resultiert die toxische Reaktion von Antigen-Antikörperkomplexen mit Komplement (Typ III nach Coombs und Gell). 
Diese beiden Typen der allergischen Reaktion laufen wohl gleichzeitig ab. Die immunologisch-entzündlichen Vorgänge zerstören das Bronchialepithel, die bronchiale Submukosa und das umliegende Lungenparenchym. Bei der unbehandelten ABPA entsteht so die zentrale (proximale) Bronchiektasie und die Fibrosierung des Lungenparenchyms, aber auch bei vermeintlich adäquater Behandlung können irreversible Organschäden eintreten.

\section{Das klinische Krankheitsbild}

Die Diagnose der ABPA wird aus Anamnese, klinischen Befunden, Hauttests, serologischen Untersuchungen und radiologischen Veränderungen gestellt.

Es hat sich bewährt, die diagnostischen Kriterien von Henderson, Patterson und Greenberger zu verwenden. Diese sind gegenüber der Beschreibung von Safirstein u. Mitarb. und Rosenberg u. Mitarb. modifiziert und etwas erweitert (Tab. 2) [8].

Von Pädiatern werden die Kriterien nach Nelson u. Mitarb. für die klinische Diagnose der ABPA bei CF häufig verwendet. Sie umfassen „wheezing“, Lungeninfiltrate, positive Sputumkultur auf A. fumigatus, Sofortreaktion im Hauttest auf A. fumigatus, erhöhtes Gesamt-IgE, spezifisches IgE und spezifisches IgG gegen A. fumigatus. Für die Diagnosestellung sollten sechs der sieben Kriterien nachweisbar sein.

Die Anzahl der positiven Kriterien und die Ausprägung sind abhängig vom Stadium der Erkrankung. Hierdurch kann die Diagnose erschwert sein und insbesondere die Abgrenzung vom allergischen Asthma bronchiale mit A.-fumigatus-Sensibilisierung Probleme bereiten. Das Gleiche gilt für die Differenzialdiagnose zwischen ABPA bei $\mathrm{CF}$ und $\mathrm{CF}$ mit A.-fumigatus-Sensibilisierung, insbesondere in der Phase der Remission der ABPA.

Wir verwenden die klinische Stadieneinteilung nach Patterson u. Mitarb. [8].

Die Erstmanifestation der ABPA wird als Stadium I bezeichnet. Bei zwei Drittel der Patienten sind akutes Auftreten von Symptomen und schubartiger Verlauf typisch, bei einem weiteren Drittel verläuft die Erkrankung in diesem Stadium subklinisch und relativ asymptomatisch. Symptome der akuten Manifestation sind die Zunahme der bronchialen Obstruktion, Fieber über mindestens 3 Tage, purulentes Sputum, Thoraxschmerzen und zähes bräunlich verfärbtes Sputum. Hämoptysen, Pleuritis sicca, Pleuraergüsse und Pneumothorax sind selten.

Stadium II der Erkrankung ist die Remission. Sie ist gekennzeichnet durch einen Rückgang des während Stadium I stark erhöhten Gesamt-IgE und des spezifischen IgG gegen A. fumigatus durch Symptomregredienz und Rückbildung der flüchtigen radiologischen Veränderungen.

Die Exazerbation der ABPA wird als Stadium III bezeichnet. Dieses Stadium ist durch die Anamnese von Stadium I zu differenzieren. Exazerbationen können auch nach Jahren der Remission unvermittelt auftreten.
Tab. 2 Kriterien der ABPA

\section{Hauptkriterien \\ 1. Asthma bronchiale/zystische Fibrose \\ 2. akute/vorbeschriebene Lungeninfiltrate \\ 3. Sofortreaktion im Hauttest auf Aspergillus fumigatus \\ 4. erhöhtes Gesamt-IgE \\ 5. Präzipitine gegen Aspergillus fumigatus \\ 6. Bluteosinophilie \\ 7. zentrale Bronchiektasen \\ 8. spezifisches IgE und spezifisches IgG gegen Aspergillus fumigatus}

\section{Nebenkriterien}

Aspergillus-Nachweis im Sputum

Expektoration von braunen Sputumpfröpfen

Spätreaktion im Hauttest auf Aspergillus fumigatus

modifiziert nach Safirstein u. Mitarb., Rosenberg u. Mitarb. und Patterson u. Mitarb.

Patterson u. Mitarb. definieren Stadium IV als kortikoidpflichtiges Asthma [9]. Heute, da viele früher systemisch-steroidpflichtige Asthmatiker unter hochdosierter Medikation mit modernen inhalativen Steroiden ohne systemische Steroide auskommen, ist diese Definition eines ABPA-Stadiums kritisch zu sehen, evtl. auch irreführend.

Das Endstadium der ABPA kann Stadium V, die Fibrose darstellen. Pulmonale Insuffizienz und Cor pulmonale führen zu schwerstem Krankheitsverlauf und schließlich zum Exitus letalis.

In den letzten Jahren wurde mit dem Begriff der „seropositiven ABPA“ (ABPA-s) ein weiteres Stadium eingeführt. Bei diesem Stadium ist die zentrale Bronchiektasie noch nicht nachweisbar (HR-CT-Untersuchung). Alle diagnostischen Möglichkeiten sollten heute deswegen genutzt werden, um insbesondere dieses frühe Stadium zu erkennen und durch konsequente Therapie eine Progredienz aufzuhalten (Tab. 3).

\section{Radiologie der ABPA}

Die radiologischen Veränderungen der ABPA können in akute (flüchtige) und bleibende Veränderungen eingeteilt werden. Akute Veränderungen sind z.B. flüchtige Lungeninfiltrate und/ oder homogene Konsolidierungen $[5,6]$.

Gloved-finger shadows (Fingerhandschuh-Schatten) sind Darstellungen erweiterter und mit Sekret gefüllter Bronchien.

Tooth-paste shadows (Zahnpasta-Schatten) sind 2 bis $3 \mathrm{~cm}$ lange Darstellungen von gering erweiterten Bronchien mit Sekretfüllung. Beide Veränderungen sind Manifestationen von mucoidimpaction, die nach Hustenattacken und/oder Behandlung mit systemischen Steroiden verschwinden können.

Weitere typische Veränderungen sind perihiläre Infiltrate, der Adenopathie nicht unähnlich, während Pleuraergüsse und Pneumothorax sehr selten gesehen werden. 
Tab. 3 Diagnostische Kriterien der ABPA in den Stadien der Erkrankung

\begin{tabular}{|c|c|c|c|c|c|c|}
\hline diagnostische Kriterien & ABPA-S & $\begin{array}{l}\text { Stadien } \\
I \\
\text { akut }\end{array}$ & $\begin{array}{l}\text { II } \\
\text { Remission }\end{array}$ & $\begin{array}{l}\text { III } \\
\text { Exazerbation }\end{array}$ & $\begin{array}{l}\text { IV } \\
\text { kortikoidpflichtiges } \\
\text { Asthma }\end{array}$ & $\begin{array}{l}v \\
\text { Fibrose }\end{array}$ \\
\hline 1. Asthma & + & + & + & + & + & + \\
\hline 2. röntgenolog. Infiltrate/Abnormalitäten/ & \pm & + & \pm & + & \pm & + \\
\hline 3. kutane Sofortreaktion auf Aspergillus fumigatus & + & + & + & + & + & + \\
\hline 5. Präzipitine auf A.f. & + & + & \pm & + & \pm & \pm \\
\hline 6. periphere Bluteosinophilie & \pm & + & - & + & \pm & - \\
\hline 7. proximale Bronchiektasie & - & + & + & + & + & + \\
\hline 8. erhöhtes Serum IgE A.f. und IgG A.f. & + & + & \pm & + & \pm & \pm \\
\hline
\end{tabular}

Eine bleibende Veränderung sehr typischer Art ist die zentrale (proximale) Bronchiektasie. Auf der konventionellen Thoraxübersichtsaufnahme können dabei proximale tubuläre Schatten oder Ringschatten gesehen werden, in der Computertomographie Siegelringzeichen oder das Perlschnurphänomen (Abb.1). Die zentrale Bronchiektasie gilt als pathognomonisch für die ABPA. Sie bevorzugt die mittleren und besonders die oberen Etagen der Lungen [5]. Eine unauffällige konventionelle Röntgenaufnahme schließt das Vorhandensein von bronchialdeformierenden Prozessen nicht sicher aus. Die radiologische Untersuchungsmethode mit der höchsten Nachweisquote ist die Highresolution-Computertomographie (HR-CT), die auch gegenüber der konventionellen Computertomographie Vorteile aufweist [5] (Tab. 4).

\section{Allergologische In-vivo- und In-vitro-Diagnostik}

Hauttests sind etablierte diagnostische Screeningverfahren. IgEvermittelte allergische Sofortreaktionen werden sowohl bei einem Asthma mit A.-fumigatus-Sensibilisierung als auch bei der ABPA beobachtet. Eine kutane Spätreaktion ist jedoch bei der ABPA häufiger zu sehen.

Wie die serologischen Untersuchungsmethoden sind Prick- und Intrakutantestung stark abhängig von Standardisierung und Konstanz der verwendeten Allergenextrakte, was besonders bei Schimmelpilzextrakten bekannterweise ein Problem sein kann (siehe unten). Wird klinisch und radiologisch eine ABPA vermutet, aber Hauttest und auch Serologie sind negativ, muss an die Möglichkeit einer durch einen anderen Pilz als A. fumigatus verursachten allergischen bronchopulmonalen Mykose (ABPM) gedacht werden [11]. Die Diagnosestellung kann hierbei aufgrund der großen Zahl infrage kommender Allergene und dem Fehlen standardisierter Allergene erhebliche Probleme bereiten.

\section{Aktueller Stand der serologischen Diagnostik}

Das Prinzip der In-vitro-Immundiagnostik basiert allgemein auf dem Nachweis zirkulierender Antikörper gegen spezifische Antigene/Allergene. Der positive Nachweis und die Konzentration verschiedener Immunglobulin-Isotypen spiegeln die immunologische Antwort des Patienten gegenüber der Exposition wider. Für eine zuverlässige und reproduzierbare serologische Diagnostik sind standardisierte Allergene erforderlich [12].

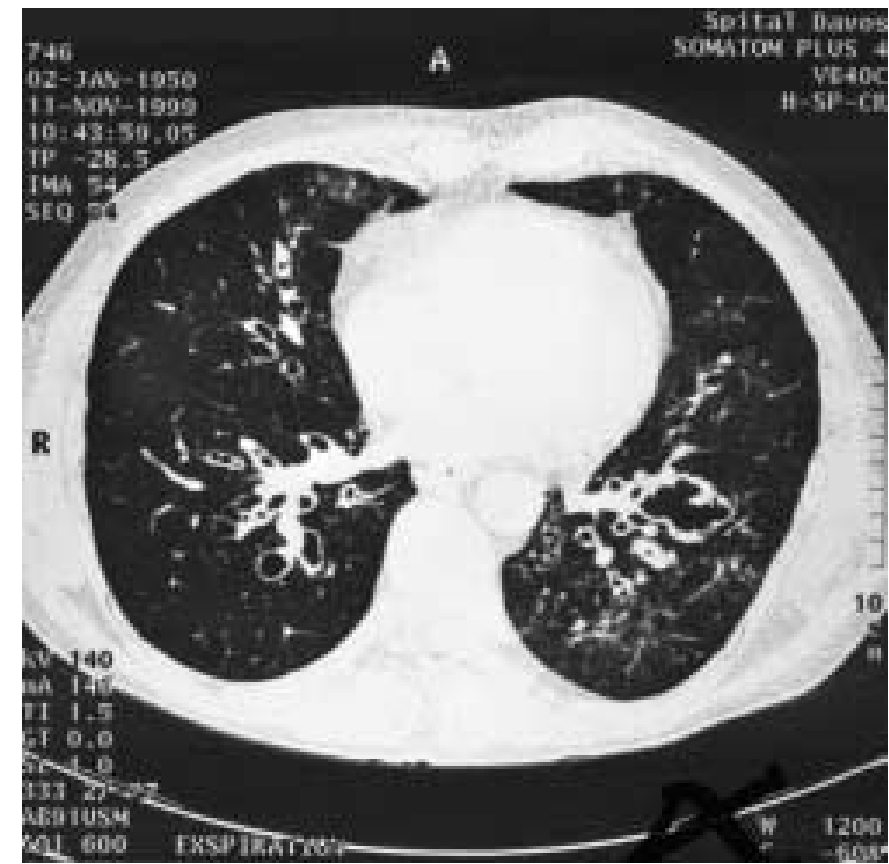

Abb. 1 Projektionsabhängige Darstellung zentraler Bronchiektasie bei ABPA.

Tab. 4 Radiologie der ABPA [5, 6]

Akute (flüchtige) Veränderungen:
- Infiltrate
- homogene Konsolidierungen
- tram-line shadows
- tooth-paste shadows
- gloved-finger shadows
- perhiläre Infiltrate
- Pneumothorax (selten)
- Pleuraerguss (selten)
Bleibende Veränderungen:
- proximale (zentrale) Bronchiektasen
- lokales Emphysem
- Lungenschrumpfung
- Fibrose


Die Herstellung standardisierter Allergenextrakte von A. fumigatus aus natürlichen Quellen wird durch eine Vielzahl verschiedener Probleme erschwert.

Ein wichtiger Grund hierfür ist die zeitabhängige Expression verschiedener Proteine während des Pilzwachstums. Eine weitere Komplikation stellt die rapide Änderung IgE-bindender Komponenten während der Fermentation dar.

A. fumigatus produziert mehr als 70 verschiedene Allergene $[13,14]$. Die Optimierung der Wachstumsbedingungen für die Produktion einer großen Zahl von Proteinen ist also nur schwer möglich. Immunoblotts mit Seren von Patienten, die an unterschiedlichen A.-fumigatus-assoziierten Erkrankungen litten, zeigten, dass die Patienten sehr unterschiedliche Konstellationen „erkannten“ (Immunopattern). Auch produziert A. fumigatus zwei bis drei verschiedene Proteasen in einer pH-Bandbreite von 2 bis 10. Diese sind aktiv in den Extrakten. Deshalb sind sogar möglicherweise weitgehend standardisierte Extrakte über eine längere Zeit sicherlich nicht stabil, was mit dem Gehalt an proteolytischen Enzymen zusammenhängt.

Die in der klinischen Routinediagnostik bisher genutzten, kommerziell erhältlichen A.-fumigatus-Gesamtextrakte sind in der In-vivo- und In-vitro-Diagnostik aus genannten Gründen nur begrenzt zuverlässig. Für die meisten Untersucher aber gibt es z.Z. noch keine echte Alternative. Aufgrund der dargestellten Komplexität und Variabilität von Schimmelpilzallergenen im Allgemeinen und A.-fumigatus-Allergenen im Besonderen ist die Standardisierung solcher Präparate eine außerordentlich anspruchsvolle und verantwortungsvolle Aufgabe. Die Lösung fordert von den Herstellern kommerzieller Extrakte intensive Forschungs- und Entwicklungsarbeiten. Die Produktion relevanter rekombinanter Allergene wird dabei in der Zukunft eine wichtige Rolle spielen.

Erst dann werden unterschiedliche Laborverfahren besser miteinander korrelieren und somit als gesicherte und reproduzierbare Kriterien für die Diagnostik gelten können.

Derzeit sollte in Fällen mit begründetem Zweifel an der Richtigkeit der Diagnose mit kommerziellen Extrakten ein Referenzlabor zu Rate gezogen werden, das mit biochemisch hochgereinigten Substanzen arbeiten kann oder noch besser das in der Lage ist, rekombinante Allergene zu verwenden.

Die Produktion biochemisch gereinigter Substanzen als Pilzkulturen wird insbesondere durch die sehr geringe Menge an hergestellten Allergen limitiert. Einige Allergene werden von Pilzen nur in sehr geringen Mengen produziert und sind nur im Immunoblott nachweisbar.

Darüber hinaus können selbst solche biochemisch hochgereinigten Substanzen mit IgE-bindenden Komponenten kontaminiert sein, was z. B. bei dem A.-fumigatus-Allergen 1 ( $r$ Aspf 1) [15] demonstriert werden und auch gezeigt werden konnte, dass sie in der Diagnostik A.-fumigatus-assoziierter Erkrankungen brauchbar sein können, sie sind für die allgemeine Routinediagnostik nicht erhältlich.
Tab. 5 Routinediagnostik-Interpretation

\begin{tabular}{|c|c|}
\hline Befund (Labor, Radiologie, Klinik) & diagnostischer Wert \\
\hline Befund (Labor, Radiologie, Klinik) & diagnostischer Wert \\
\hline Asthma/CF & ABPA möglich \\
\hline Eosinophilie & ABPA möglich \\
\hline flüchtige pulmonale Infiltration & ABPA möglich \\
\hline zentrale Bronchiektasie & ABPA fast sicher \\
\hline Serologie & $\begin{array}{l}\text { diagnostischer Score der kompletten } \\
\text { Serologie }\end{array}$ \\
\hline 1. Präzipitine gegen A. fumigatus pos. & 4 Tests positiv = Diagnose sicher \\
\hline $\begin{array}{l}\text { 2. A. fum.-IgE-Antikör- } \\
\text { per }>2 \text { fach-Asthmakontrolle }\end{array}$ & $\begin{array}{l}3 \text { Tests positiv = Diagnose sehr wahr- } \\
\text { scheinlich }\end{array}$ \\
\hline $\begin{array}{l}\text { 3. A. fum.-IgG-Antikör- } \\
\text { per }>2 \text { fach-Asthmakontrolle }\end{array}$ & 2 Tests positiv = Diagnose möglich \\
\hline 4. Gesamt-Serum-IgE $>1000$ & \\
\hline
\end{tabular}

Die derzeit meist verwendete serologische Routinediagnostik ist in Tab. 5 zusammengefasst. Es sei an dieser Stelle darauf hingewiesen, dass negative A.-fumigatus-Präzipitine unter einer systemischen Steroidtherapie der ABPA vorkommen können. Sehr typisch für die ABPA ist der Abfall des Gesamt-IgE um 50 bis $75 \%$ nach einer hochdosierten Steroidtherapie. Sind alle vier Tests positiv, kann die Diagnose als sicher wahrgenommen werden, sind drei der vier Tests positiv, ist die Diagnose sehr wahrscheinlich; bei zwei positiven Tests ist die Diagnose möglich [10].

\section{Die Verwendung rekombinanter A.-fumigatus-Allergene für die Diagnostik der ABPA}

Im Schweizer Institut für Asthma- und Allergieforschung (SIAF) gelang es zuerst, das A.-fumigatus-Hauptallergen 1 (rAspf 1) zu sequenzieren und zu klonen und sowohl in vitro als auch in vivo zu prüfen [15]. rAspf 1 ist ein Protein aus der Familie der Ribotoxine mit einem Molekulargewicht von $18 \mathrm{kD}$. Es zeigt zytotoxische Eigenschaften und ist einer der potentesten und spezifischen Inhibitoren der eukaryotischen Translation. Es führt zu einer enzymatischen Spaltung der 28rRNA und damit zur irreversiblen Inaktivierung der Ribosomen in der Zelle. Das klonierte rAspf 1-Gen wird von E. coli mit einer Ausbeute von $15 \mathrm{~g}$ reinem Protein auf 1001 Fermentationsmedium produziert und ist damit in praktisch unbegrenzter Menge verfügbar.

Das Protein wurde bei allergischen Asthmatikern mit A.-fumigatus-Sensibilisierung, Patienten mit Mukoviszidose und Patienten mit atopischer Dermatitis auf seine diagnostische Aussagefähigkeit untersucht. Für Patienten mit allergischem Asthma bronchiale und A.-fumigatus-Sensibilisierung, Patienten mit ABPA, Patienten mit Mukoviszidose und A.-fumigatus-Sensibilisierung ist rAspf 1 ein Hauptallergen [15]. Die Entwicklung eines neuen Klonisierungssystems (pJuFo) [16] führte zu einer schnelleren Isolierung und Charakterisierung von A.-fumigatus-Allergenen. Vier dieser Allergene wurden immobilisiert und auf ihren routinemäßigen Einsatz im Immuno-CAP-System untersucht [17]. Unsere angeführten Untersuchungen haben gezeigt, dass rekombinante Allergene für die In-vitro- und In-vivo-Diagnostik allergischer Erkrankungen geeignet sind. Die exakte Übereinstim- 
mung zwischen Hauttestergebnissen und Serologie [18] zeigt die Möglichkeiten und das Potenzial rekombinanter Allergene in der Diagnostik allergischer Erkrankungen.

\section{Welche A.-fumigatus-Allergene sind für die Diagnose und Differenzialdiagnose der ABPA von entscheidender Bedeutung?}

Wie oben angeführt, ließ die Verwendung von rAspf 1 allgemein eine sichere Unterscheidung zwischen ABPA und A.-fumigatusSensibilisierung (z.B. bei Asthma) nicht zu, da Seren von ABPA-Patienten und auch Nicht-ABPA-Patienten IgE-Antikörper gegen Aspf 1 enthalten können [15].

Nachdem die gentechnische Produktion weiterer A.-fumigatusAllergene gelungen war, dehnten wir die in-vivo- und in-vitroUntersuchungen zunächst auf rAspf 3 aus. rAspf 3 ein 18,5 kD peroxisomales Protein ist ein Major-Allergen von A. fumigatus. 84\% der Asthmapatienten mit A.-fumigatus-Sensibilisierung zeigten eine positive Pricktestreaktion. Die In-vivo-Testung und die In-vitro-Untersuchung mit Immuno-CAP zeigten eine $100 \%$ ige Korrelation. Es gab keine falsch positiven oder falsch negativen Resultate.

Eine Diskriminierung von ABPA und A. fumigatus-sensibilisierten Allergikern ist jedoch wie schon mit rAspf 1 auch mittels rAspf 3 nicht möglich $[18,19]$.

\section{rAspf 4 und rAspf 6 ermöglichen die Differenzialdiagnose von ABPA mit einer Spezifität von $100 \%$ und einer Sensitivität von über $90 \%$}

Im Unterschied zu den oben erwähnten Untersuchungen mit rAspf 1 und rAspf 3 zeigten die serologischen Tests unter Benutzung von rAspf 4 , einem $34 \mathrm{kD}$-Protein unbekannter biologischer Funktion und Verwendung von rAspf 6, der Mangan-Superoxiddismutase, eine klare Trennung zwischen den Patientengruppen. Spezifisches Serum-IgE gegen beide Allergene konnte nur bei den ABPA-Patienten nachgewiesen werden [17,18]. Abb. 2.

So ist die Diagnose der ABPA allein durch diese spezifische serologische Untersuchung auch in der Remissionsphase der Erkrankung möglich.

Bei Mukoviszidose, bei der eine Unterscheidung zwischen ABPA und A.-fumigatus-Sensibilisierung (ohne ABPA) besonders schwierig und besonders bedeutsam ist, reichen die Allergene rAspf 4 und rAspf 6 aus, um eine serologische Diagnose mit nahezu $100 \%$ iger Sensitivität zu stellen.

Die beschriebenen Aspergillus-Allergene gehören zwei verschiedenen Gruppen an: sekretierten und zytoplasmatischen Proteinen. Während die sekretierten Allergene (z.B. rAspf 1 und rAspf 3) sowohl von IgE-Antikörpern A. fumigatus-sensibilisierter Individuen mit ABPA als auch ohne ABPA erkannt werden, werden die nicht sekretierten Allergene ausschließlich von IgE von ABPA-Patienten gebunden [18-20].

So ist zum Beispiel rAspf 6 (Mangansuperoxiddismutase) ein strikt intrazelluläres Protein, wie auch rAspf 4. Beide Proteine werden vom Schimmelpilze nicht sekretiert und wirken so nicht als Aero-Allergen, was die negativen Resultate bei den A. fumiga-
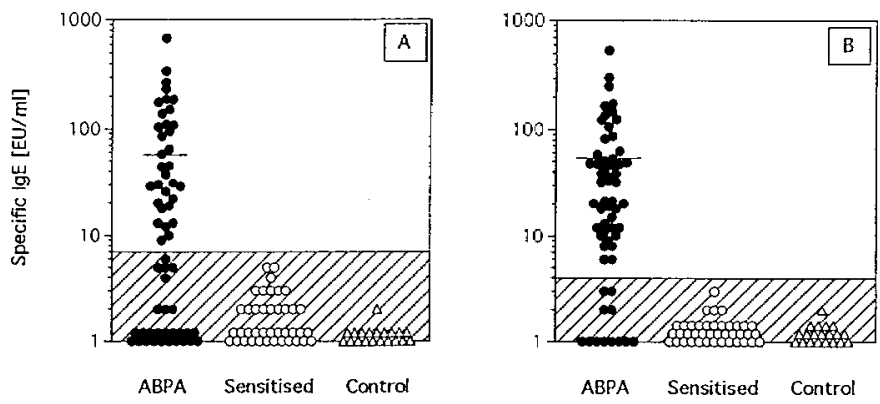

Abb. 2 Spezifische IgE-Antikörper gegen rAspf 6 (A) und rAspf 4 (B) in Seren von gesunden Kontrollindividuen und A. fumigatus-sensibilisierten Asthmatikern mit oder ohne ABPA. Die IgE-Bindung gegenüber dem Allergen wurde mit einem antigenspezifischen ELISA bestimmt und verglichen mit der IgE-Bindung eines Referenzserums, das auf $100 \mathrm{EU} / \mathrm{ml}$ eingestellt ist. Die Mittelwerte sind mit Linien dargestellt. Die gestrichelte Fläche repräsentiert den Cut-off-Wert, der durch Hauttests ermittelt wurde. Die Daten zeigen, dass diese beiden Allergene hochspezifisch sind für Seren von Patienten mit ABPA. Von den $60 \mathrm{~Pa}-$ tienten mit ABPA zeigten 48 (80\%) eine IgE-Antwort gegenüber rAspf 4, 33 (55\%) gegenüber rAspf 6 und 54 (90\%) erkannten wenigstens eines der beiden Allergene. Damit erreicht die Sensitivität für die Diagnose der ABPA 90\% und die Spezifität 100\%.

tus-sensibilisierten Asthmatikern erklärt. Bei den ABPA-Patienten kommt es jedoch zum Wachstum des Pilzes in den Bronchien und der Lunge. Die zelluläre Immunabwehr des Wirtsorganismus führt dann zur Zerstörung der Pilzstrukturen und damit zur Exposition gegenüber nicht sekretierten Antigenen/Allergenen.

Die rekombinanten Aspergillus-Allergene 1, 2, 3, 4 und 6 sind jetzt seit 2000 kommerziell im CAP-RAST-FEIA-System (Fa. Pharmacia) erhältlich und stehen für die Routinediagnostik zur Verfügung.

\section{Therapie der ABPA}

Die beschriebene Stadieneinteilung hilft bei der Therapieplanung. Kortikosteroide (Prednison/Methylprednisolon) sind Mittel der Wahl in der Behandlung akuter Lungeninfiltrate in Stadium 1 (akute Erstmanifestation) und Stadium III (Exazerbation) und in Stadium IV (kortikoidpflichtiges Asthma). Eine Dosis von $0,5 \mathrm{mg} / \mathrm{kg} / \mathrm{Tag}$ wird allgemein initial über 2 Wochen empfohlen [20].

Bei den meisten Patienten wird damit eine Remission eingeleitet, die Infiltrate verschwinden, die klinischen Symptome sind unter Kontrolle und das Gesamt-IgE beginnt drastisch zu fallen. Gewöhnlich sind 2 bis 4 Wochen einer täglichen Steroidtherapie in angepasster Dosierung zwischen $0,25 \mathrm{mg} / \mathrm{kg} / \mathrm{Tag}$ und $0,5 \mathrm{mg} / \mathrm{kg} /$ Tag ausreichend. Neu diagnostizierte Fälle in Stadium IV und besonders Stadium V können jedoch initial höhere Dosierungen erforderlich machen.

Falls eine adäquat durchgeführte Steroidtherapie nicht innerhalb von 4 bis 6 Wochen zum Rückgang der Lungeninfiltrate bzw. anderer flüchtiger Lungenveränderungen oder Besserung der klinischen Symptomatik führt, muss zum einen an eine mangelhafte Compliance seitens des Patienten, zum anderen an eine die ABPA komplizierende zusätzliche Lungenerkrankung gedacht werden. 
Eine bakterielle Pneumonie kann zusätzlich vorliegen und erfordert neben der Steroidtherapie eine gezielte Antibiose.

Ein komplettes Programm zur optimalen Sekretdrainage ist bei allen ABPA-Patienten wie bei anderen Krankheitsbildern mit Bronchiektasie erforderlich.

Röntgenologische Kontrollen sind nach Therapieeinleitung zumindest nach 2 und 4 Wochen notwendig. Falls keine ausreichende Rückbildung der flüchtigen röntgenologischen Veränderungen zu verzeichnen ist, muss die Kortikosteroiddosis erhöht werden. Wie das Gesamt-IgE fällt auch das spezifische IgG-A. fumigatus bei einer erfolgreichen Remissionsinduktion deutlich ab.

Ein weiterer Schritt zur Behandlung der ABPA ist die AspergillusEradikation aus dem Bronchialsystem zur Reduktion der fungalen Belastung und der mit ihr verbundenen chronischen antigenen Stimulation. In einer doppelblind randomisierten Studie von Stevens u. Mitarb. [22] konnte bei 28 Patienten über 16 Wochen ein Vorteil einer antimykotischen Therapie mit $2 \times 200 \mathrm{mg}$ Itraconazol pro Tag bei steroidpflichtiger ABPA bei gleicher Toxizität nachgewiesen werden. Ein Ansprechen auf die antimykotische Therapie wurde u. a. über eine Dosisreduktion der Steroiddauermedikation von mind. 50\% sowie einen Abfall des Gesamt IgE von mind. 25\% definiert. Zu einem vergleichbaren Ergebnis kamen jüngst Wark u. Mitarb. [23], die zudem einen Abfall des spezifischen IgG-Serumspiegels, der Sputumeosinophilie sowie der Exazerbationshäufigkeit unter Itraconazoltherapie sahen. Für die Wirksamkeit des Itraconazol spricht darüber hinaus die Studie von Salez u. Mitarb. [24] an einem kleinen Kollektiv von 14 Patienten, die zunächst in einer 2-jährigen Referenzperiode mit Steroiden (inhalativ/systemisch) behandelt wurden, und im Anschluss täglich $200 \mathrm{mg}$ Itraconazol über einen Zeitraum von 12 Monaten erhielten. Während in der Referenzzeit keine signifikante klinische, immunologische und funktionelle Verbesserung gesehen wurde, waren unter Itraconazol-Gabe die Bluteosinophilie, der Gesamt-IgE-Spiegel und die präzipitierenden Antikörper gegen A. fumigatus Antigen signifikant rückläufig. Das spezifische IgE gegen A. fumigatus blieb unbeeinflusst [24]. Die Dosis des Kortikosteroids konnte bei allen Patienten signifikant reduziert bzw. bei 7 Patienten das Steroid sogar vollständig abgesetzt werden [24].

Zur Zeit fehlen noch randomisierte Studien zur Frage der Behandlungsdauer und zum weiteren Vorgehen bei Rezidiven.

Zusammenfassend stellen Kortikosteroide die Basistherapie der ABPA dar, während es für den adjuvanten Einsatz des Itraconazol zunehmende Evidenz gibt, die Indikation aber weiterhin sehr sorgfältig zu stellen ist. Zu beachten bleiben in Zeiten zunehmenden Kostendrucks auch Mehrkosten von über $2000 €$ für eine 16-wöchige Therapie mit $2 \times 200 \mathrm{mg}$ Itraconazol.

Wichtig erscheint, die Patienten über Möglichkeiten einer weitestgehenden Allergenkarenz aufzuklären und dezidiert auf negative Einflüsse hinzuweisen. Derzeitig übliche Methoden der Abfallaufbewahrung (Biotonnen) und Kompostierung können die Allergenbelastung durch Aspergillus und andere Schimmelpilze deutlich vermehren. In komplizierten Fällen der ABPA mit häufigen Exazerbationen und/oder rascher Progredienz kann nur eine Behandlung in spezialisierten Einrichtungen in Höhenlagen über $1500 \mathrm{~m}$ absolute Allergenkarenz gewährleisten und damit den therapeutischen Durchbruch bringen. [21].

Die Zukunft wird zeigen, ob eine bessere Überwachung der asymptomatischen Patienten mittels ,immunologischem Fingerabdruckes“" (Immunoprint) möglich sein wird (Verwendung der „rekombinanten Serologie“).

Die Frühdiagnostik der ABPA, möglichst in Phasen noch ohne bleibende Veränderungen von Bronchialsystem und Lungenparenchym, die adäquate und konsequente Therapieeinleitung und Überwachung der Remissionsphase werden die progrediente Entwicklung der Endstadien verhindern helfen.

\section{Literatur}

${ }^{1}$ Hinson KFW, Moon AJ, Plummer NS. Bronchopulmonary aspergillosis. Thorax 1952; 7: 317-333

2 Slavin RG, Stanczyk DJ, Lonigro AJ et al. Allergic bronchopulmonary Aspergillosis - A North American rarity. Am J Med 1969; 47: 306 - 313

${ }^{3}$ Leser C, Kauffmann HF, Virchow C et al. Specific serum immunopatterns in clinical phases of Allergic Bronchopulmonary Aspergillosis. J Allergy Clin Immunol 1992; 90: 589-599

${ }^{4}$ Moser M, Crameri R, Brust E et al. Diagnostic value of recombinant Aspergillus fumigatus allergen I/a for skin testing and serology. J Allergy Clin Immunol 1994; 93: 1 - 11

${ }^{5}$ Menz G, Virchow C. Röntgendiagnostik bei Allergischer bronchopulmonaler Aspergillose unter Berücksichtigung der Computertomographie. Atemw und Lungenkr 1987; 13: 140-144

${ }^{6}$ Mintzer RA, Rogers LF, Kruglik GD. The spectrum of radiologic findings in Allergic Bronchopulmonary Aspergillosis. Radiology 1978; 127: $301-307$

${ }^{7}$ Nikolaizik WH, Brueton MJ, Warner J. Aspergillus allergy and ABPA in cystic fibrosis. Pediatr Allergy Immunol 1991; 2: 83-86

8 Patterson R, Greenberger PA, Radin RC et al. Allergic bronchopulmonary Aspergillosis: staging as an aid to management. Am Int Med 1982; 96: 286-291

${ }^{9}$ Patterson R, Greenberger PA, Lee TM et al. Prolonged evaluation of patients with corticosteroid-dependent asthma stage of Allergic bronchopulmonary aspergillosis. J Allergy Clin Immunol 1987; 80: $663-668$

${ }^{10}$ Greenberger PA, Miller TP, Robert M et al. Allergic Bronchopulmonary Aspergillosis in patients with and without evidence of bronchiectasis. Ann Allergy 1993; 70: 333-338

${ }^{11}$ Matthieson AM. Allergic bronchopulmonary-disease caused by fungi other than aspergillus. Thorax 1981; 36: 719

12 Borga A. Allergens of Aspergillus fumigatus. Stockholm: PhD Thesis, Karolinkska Institute, Repro Print AB, 1990

${ }^{13}$ Crameri R, Walter G. Selective enrichment and high-throughput screening of phage surface-displayed CDNA libraries from complex allergenic systems. Combin Chem High Throughput Screen 1999; 2: $63-72$

${ }^{14}$ Kurup VP, Kumar A. Immunodiagnosis of Aspergillosis. Clin Mikrobiol Rev 1991; 4: 439-456

${ }^{15}$ Moser M, Crameri R, Menz G et al. Cloning and expression of recombinant Aspergillus fumigatus allergen 1 (rAspf1/a) with IgE binding and type I skin test activity. J Immunol 1992; 41: 454-460

${ }^{16}$ Crameri R, Blaser K, Menz G. Rekombinante Allergene und ihr Potential für die allergologische Diagnostik. Pneumologie 1996; 6: $387-393$

${ }^{17}$ Crameri R, Lidholm J, Grönlund $\mathrm{H}$ et al. Automated specific IgE assay with recombinant allergens: evaluation of the recombinant Aspergillus fumigatus allergen 1 in the Parmacia CAP System. Clin and Experimental Allergy 1996; 26: 1411 - 1419

${ }^{18}$ Hermann S, Menz G, Ismail C et al. Skin test reactivity to two recombinant Aspergillus fumigatus allergens in A Aspergillus fumigatus - sensitised asthmatic subjects allows diagnostic separation of allergic 
bronchopulmonary aspergillosis from fungal sensitisation. J Allergy Clin Immunol 1999; 104: 478-484

${ }^{19}$ Crameri R, Hemmann S, Ismail C et al. Disease-specific recombinant allergens for the diagnosis of allergic bronchopulmonary aspergillosis. Int Immunol 1998; 10: 1211 - 1216

${ }^{20}$ Menz G, Ismail C, Crameri R. Die allergische bronchopulmonale Aspergillose. Pneumologie 2000; 54: 375-384

${ }^{21}$ Ismail C, Crameri R, Menz G. Serologische und klinische Verlaufsparameter der Allergischem bronchopulmonalen Aspergillose (ABPA). Eine Längsschnittuntersuchung. Atemw- und Lungenkr 1995; 11: 573

22 Stevens DA, Schwartz HJ, Lee JY et al. A randomized trial of itraconazole in allergic bronchopulmonary aspergillosis. N Engl J Med 2000; 342 (11): $757-762$

${ }^{23}$ Wark PAB, Hensley MJ, Saltos $\mathrm{N}$ et al. Anti-inflammatory effect of intraconazole in stable allergic bronchopulmonary aspergillosis: A randomized controlled trial. J Allergy Clin Immunol 2003; 111: 952 - 7

${ }^{24}$ Salez F, Brichet A, Desurmont S et al. Effects of itraconazole therapy in allergic bronchopulmonary aspergillosis. Chest 1999; 116 (6): $1665-1668$
Bereits publizierte Beiträge zu dieser Serie:

1 Pulmonale Lymphangioleiomyomatose. Pneumologie 2002; 56: 309-315

${ }^{2}$ Alveolarproteinose. Pneumologie 2002; 56: $448-456$

${ }^{3}$ Idiopathisch eosinophile Pneumonien. Pneumologie 2002; 56: $621-630$

${ }^{4}$ BOOP. Pneumologie 2002; 56: 798-806

${ }^{5}$ Pulmonale Langerhans-Zell-Histiocytosis. Pneumologie 2003; 57: $159-165$

${ }^{6}$ RB-ILD. Pneumologie 2003; 57: $278-287$

\section{Buchbesprechung}

\section{Kosmologie für die Westentasche \\ Rudolf Kippenhahn}

Piper Verlag GmbH, München 2003, 127 S. , zahlreiche Abb., Register. Geb. $€$ 9,90; CHF 17,40. ISBN 3-492-04497-2

In Jahrmillionen der Evolution haben unsere Gehirne auch Fähigkeiten entwickelt, die für das Überleben völlig ohne Bedeutung sind: wir konzipieren philosophische (nach R. von Wittgenstein „unsinnige“) Konstrukte, erobern beim Bergsteigen das völlig nutzlose, aber oft tödliche „Gipfelglück“ (R. Messmer) und können auch abstrakte mathematische Gedankengebäude errichten. Für den „Lebenskampf“ sind sie alle ohne Gewicht (R. Kippenhahn).

Doch die „Fähigkeiten“ ermöglichen es uns, u.a. auch das unermessliche Weltall mit seiner Materie wie auch die „Miniwelt“ der Atome intellektuell zu verstehen.

Aber können wir es uns tatsächlich vorstellen, dass das Licht von Sternen (=Sonnen) aus der grenzenlosen Weite des Raums uns erst nach Millionen von Jahren erreicht - und dass ein Quark kleiner als ein Atom ist? Dass das Weltall einst sehr viel kleiner gewesen und erst durch quantenphysikalische Prozesse aus dem
„Nichts“ entstanden sei - dem „Urknall (Big Bang)“? Dass der endlose Weltraum weder Mittelpunkt noch Grenzflächen habe, von den Physikern jedoch als „gekrümmt“ bezeichnet wird? Und dass die im Raume befindliche Materie sich immer weiter und desto schneller voneinander entferne und so einmal die bisher ultimative Geschwindigkeit des Lichts (300000 km/sec) „überholen“ müsste?

Auf solche verwirrende Fragen gibt Verf. in seinem „Westentaschenbuch“ verständliche Erklärungen: von der Astronomischen Einheit (Abstand von Sonne zur Erde=1 AE) bis zur „Ursuppe“; von der „Milchstraße“ bis zu den Tausenden anderer Galaxien und deren Formen (z.B. Spiral- und elliptischen Galaxien); von der „kosmischen Hintergrundstrahlung“ (Penzias/Wilson 1964), der „kalten Wärmestrahlung“ von $-270^{\circ} \mathrm{C}$ (als einem Beweis für den „Urknall“); von „rhythmischen Sternen als Standardkerzen“ bis zu Radiosternen, die keine Sterne sind; vom Doppler-Effekt bis zur Dunklen Materie; von Teilchen und Antiteilchen etc. etc. Für den am „Unendlichen“, dem für uns „Ewigen“ Interessierten ein lesenswertes Buch, das quasi „per Anhalter durch das Weltall“ führt.

Heinz S. Fuchs, Bad Godesberg 